\title{
A Network Coding-Based Reliable Multicast Routing Protocol for Efficient Data Delivery in Mobile Ad-hoc Wireless Networks
}

\author{
${ }^{1}$ Le The Dung and ${ }^{2}$ Beongku An \\ ${ }^{1}$ Dept. of Electronics and Computer, Engineering in Graduate School, Hongik \\ University, Korea \\ ${ }^{2}$ Dept. of Computer and Information Communications Engineering, \\ Hongik University, Korea \\ Ithedung_hcmut@yahoo.com,beongku@hongik.ac.kr \\ Abstract
}

\begin{abstract}
Network coding is an emerging approach which is currenty applied to wireless networks to improve network throughput and other performance. However, in mobile ad-hoc wireless networks, because of dynamic network topology, network coding may not improve network throughput if destination nodes fail to retrieve original packets due to lack of necessary information to decode coded packets. In this poper, we propose a Network Coding based Reliable Multicast Routing Protocol, called CRMP, in moblle ad-hoc wireless networks. Our proposed CRMP has the following novefeatures: i) it builds a stable mesh structure to improve coding and decoding opponynities, iv uses random linear network coding to simplify coding algorithm, iii) it has a local route repairing mechanism to prevent decoding failures caused by local route breaks. CRMP isespecially well-suited for reliable, high speed multimedia applications. The performance of our proposed CRMP is evaluated via different scenarios. The results show that CRMPKoutperforms previously proposed multicast routing.
\end{abstract}

Keywords: Mobile ad-hoc wireless networks, multicast routing, stable mesh structure, linear network eoding, decoding failure, local route repair

\section{Introduction}

Network coding is a coding conception which is first proposed in [1]. It is a highly efficient transmission iechnique which has fundamentally changed the way how data packets are delivered in communication networks. Unlike traditional store-and-forward packet delivery technique, network coding implements store, code, and forward approach, where each node stores incoming data packets in its buffer, combines information in different data packets when coding condition appears, then sends coded packets it is proved in the literature that network coding allows the communication to achieve higher throughput.

Prior works in network coding can be classified into three categories such as theoretical approaches [1-3], testbed approaches [4-6], and simulation approaches [711]. The theoretical approaches extend information theory in wired networks for wireless networks to calculate bound values of network throughput and end-to-end delay. Testbed approaches use implementations and experiments with specific hardware devices to demonstrate the throughput gain in practice. The simulation approached design and evaluate network coding protocols or network coding based routing protocols. This paper belongs to the third category.

The rests of this paper are organized as follows. In Section 2, we present previous network coding based routing protocols in mobile ad hoc wireless networks. In Section 
3 , the benefits and challenges of network coding are discussed. In Section 4, we describe in detail our proposed network coding based reliable multicast routing protocol. In Section 5, we evaluate its performance in different settings network parameters and compare with (i) ODMRP [12], a traditional multicast routing protocol without network coding and (ii) CodeCast [7], a previously proposed network coding based multicast routing protocol. Finally, Section 6 concludes the paper.

\section{Related works}

In this section, we will give a brief survey of previous works on designing and evaluating network coding protocols by using simulation method. In [7], a network coding based ad hoc multicast protocol, called CodeCast, is proposed However, CodeCast suffers from decoding failure because of link break due to node that will be mentioned in Figure 1(a). The authors in [8] use both opportunistic routing and network coding to improve network performance. Since link's ETX metric and Steiner tree are used in [8], it requires a lot of control overheads to maintain network connectivity. The authors in [9] apply network coding to reduce overall oxerheads in multicast data delivery. Then, a hierarchical network topology is used to deal with scalability issue in large scale mobile ad hoc networks. In [10] the authors improve the performance of ODMRP by applying network coding. They gnly apply network coding to Join Request packets. Thus, the coding efficiency is not high. In [11], a multicast routing protocol for network coding (NCMRP) is proposed by bringing network coding into multicast with a few minor changes to the protofo packet formats of distance vector multicast routing protocol (DVMRP). The basicidea of NCMRP is that the source finds multiple disjoint paths from the source to each multicast destination node, and the multicast topology is deployed for network coding. However in these previous works no algorithms to construct robust network topology against node mobility as well as no mechanisms to prevent network decoding failures at multicast destination nodes due to node mobility are mentioned With motivations by all issues in previous works, in this paper we propose a practica) Network Coding based Multicast Routing, called CRMP for short, in mobile ad-hoc wireless networks with a stable network structure, a novel packet coding-and-forwarding decision, and a local route repairing mechanism to improve network throughput while reducing network congestion for route maintenance.

\section{Background and Motivations}

\subsection{The Benefit of Network Coding in Multicasting}

Network coding helps to reduce the number of data transmissions (i.e. network congestion) in multicasting, especially in vulnerable network topologies such as mobile ad-hoc wireless networks. First, let us illustrate how network coding can reduce network congestion during packet transmission by using Figure 1(a).

Source node $\mathrm{S}$ wants to multicast two data packets $\mathrm{P}_{1}$ and $\mathrm{P}_{2}$ to destination node $\mathrm{D}_{1}$ and $\mathrm{D}_{2}$. Without using linear network coding, a total of 10 transmissions are needed as in Figure 1(a). By using linear network coding, source node $\mathrm{S}$ can also multicast the same two data packets to $D_{1}$ and $D_{2}$ as follows. Firstly, source node $S$ sends two linearly combined packets. Then, if each forwarding node receives two different incoming packets it will recombine those packets with different coding coefficients. Otherwise, it simply forwards the previously received code packet. Destination node can retrieve data packets $\mathrm{P} 1$ and $\mathrm{P} 2$ if they receive two coded packets with linear 
independent coefficients. In summary, with network coding, a total of 6 transmissions are needed compared with 10 transmissions in the case of without linear network coding.

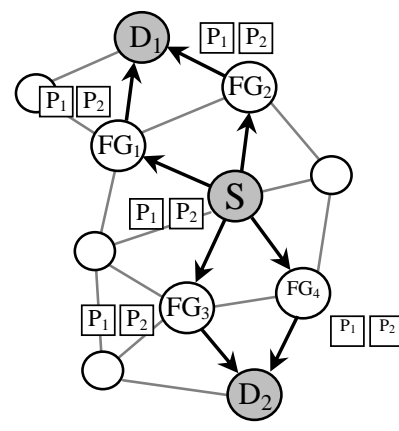

(a)

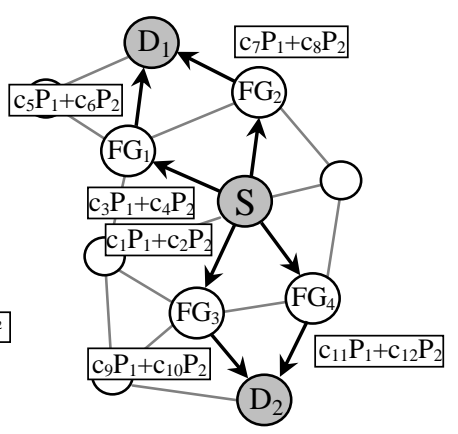

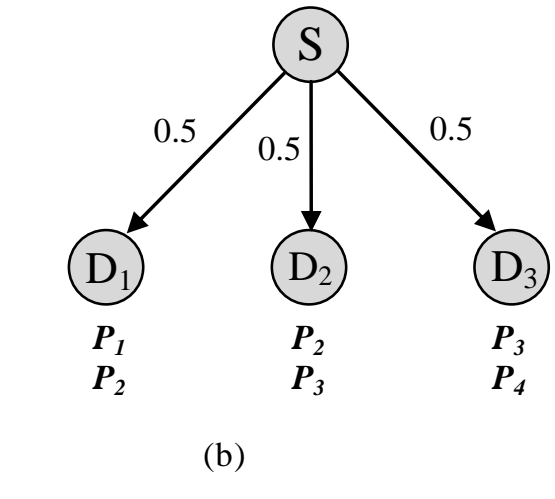

Figure 1. The Benefits of Network Coding in Multicast Routing during (a) Packet Transmission and (b) Packet Retransmission

Second, we will illustrate how network coding can reduce network congestion during packet retransmission by using Figure 1 (b). Soure node $S$ wants to multicast four packets $\mathrm{P}_{1}, \mathrm{P}_{2}, \mathrm{P}_{3}$, and $\mathrm{P}_{4}$ to three destination nodes $\mathrm{D}_{1}, \mathrm{D}_{2}, \mathrm{D}_{3}$. Due to link quality problem caused by node mobility and network congestion, destination nodes cannot receive all four packets. For example, souree node can only deliver data packets to each destination nodes with delivery probability of $50 \%$. Since packet reception at each destination node is independent, eadh destination node receives different packet as in Figure 1(b). Without network coding, source node $S$ has to sequentially send all four packets again (i.e $\mathrm{R}_{1}$ for $\mathrm{D}_{2}$ and $\mathrm{D}_{3}, \mathrm{P}_{2}$ for $\mathrm{D}_{3}, \mathrm{P}_{3}$ for $\mathrm{D}_{1}$, and $\mathrm{P}_{4}$ for $\mathrm{D}_{1}$ ) so that all destination nodes can receive all four packets. However, if network coding is used, source node $\mathrm{S}$ just needs to tyansmit two linear combinations, e.g. $\mathrm{P}_{1}{ }^{\prime}=\mathrm{P}_{1}+\mathrm{P}_{2}+\mathrm{P}_{3}+$ $\mathrm{P}_{4}$ and $\mathrm{P}_{2}{ }^{\prime}=\mathrm{P}_{1}+2 \mathrm{P}_{2}+3 \mathrm{P}_{3}+4 \mathrm{P}_{4}$. After receiving those two coded packets, destination nodes can retrieverissing packets, saving the source nodes two transmissions.

\subsection{The Challenge of Network Coding: Decoding Failure}

We observe an important fact that in dynamic networks like mobile ad-hoc networks netwo k coding may not increase network throughput or may degrade network performance if multicast destination nodes may not receive enough coded packets to retrieve original packets. Let us illustrate that issue by using Figure 2 .

Due to node mobility, the wireless link $\mathrm{FG}_{3}-\mathrm{D}_{2}$ is broken. Thus, destination node $\mathrm{D}_{2}$ cannot receive two linearly independent coded packets to recover data packets $\mathrm{P}_{1}$ and $\mathrm{P}_{2}$. This issue can be solved with local route repairing mechanism in our proposed network coding based reliable multicast routing. 


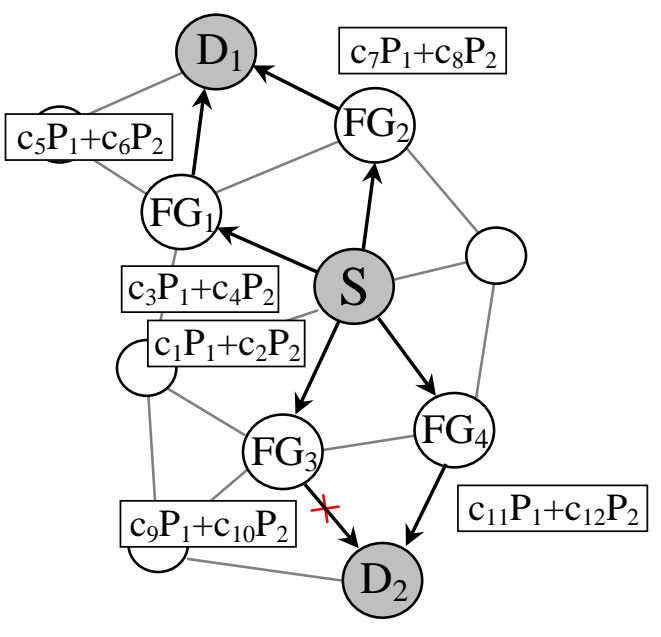

Figure 2. The Impact of Route Break on the Performance of Linear Network Coding

\section{The Proposed Network Coding based Reliable Multicast Routing}

In this section, we present in detail the arehitecture of our proposed network coding based reliable multicast routing, calledCERMP, which includes the format of data packet and step-by-step routing and coding âtgorithm?

\subsection{Packet Format}

Our proposed network coding based reliable multicast routing protocol inserts coding header in each coded data packet as in Frgure 3.

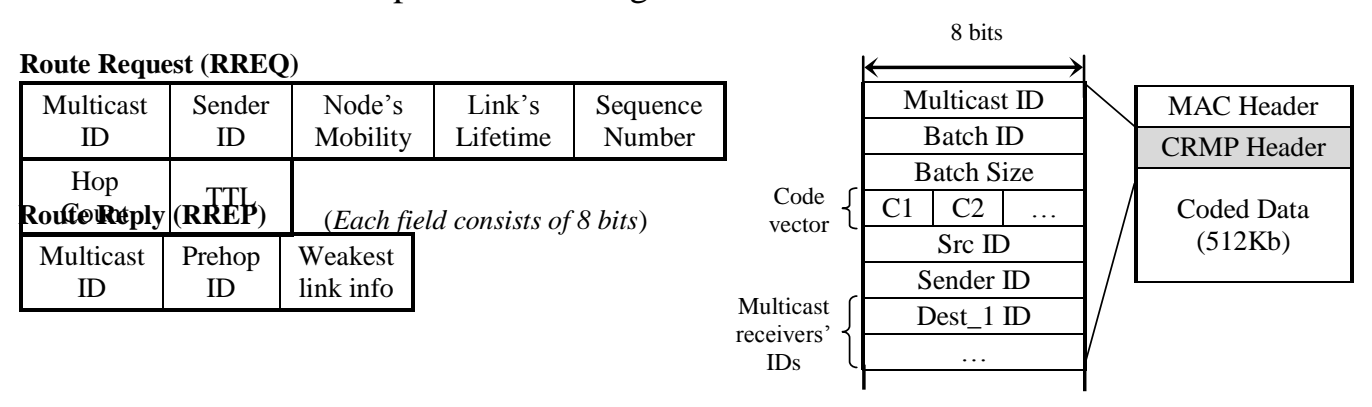

(a)

(b)

\section{Figure 3. The Format of (a) Control Overheads and (b) Data Packet in our Proposed Network Coding based Multicast Routing Protocol}

Multicast ID is the identified number of each multicast group. To perform linear network coding, source node divides data packet stream into batches. Each batch contains $M$ data packets. Batch Size is set to $M$ which can be changed. A data packet $p_{k}$ is said to be in the batch (Batch ID, Batch Size) if Batch ID $\leq k \leq$ Batch ID + Batch Size. Code vector has vector size equal to the value in Batch Size. All elements in code vector are random integer values from 1 to 9 . Src ID is the ID of source node in multicast group. Sender ID is the ID of node sending the data packet. The list of multicast receivers' IDs is also specified in code header. 


\subsection{Network Coding based Reliable Multicast Routing Protocol}

Our proposed network coding based reliable multicast routing protocol establishes mesh structure as in ODMRP [12] to exploit spatial diversity of forwarding nodes in mesh, which helps to improve coding and decoding opportunities. We also apply our previous work [13] to provide stable mesh structure for better network coding performance. Moreover, a local route repairing mechanism is used to prevent decoding failure at multicast destination nodes. Figure 4(a) illustrates the basic concept of our proposed CRMP while Figure 4(b) presents the algorithm to prevent decoding failure at multicast destination nodes. As we can see in Figure 4(b), CRMP can repair local route breaks by using Route Expore (RtE) packet and Route Construct (RtC) packet to prevent decoding failure at destination node. RtE and RtC have the samp format as RREQ and RREP, respectively, except that their TTLs are set to two hops to prevent them from flooding throughout the network.

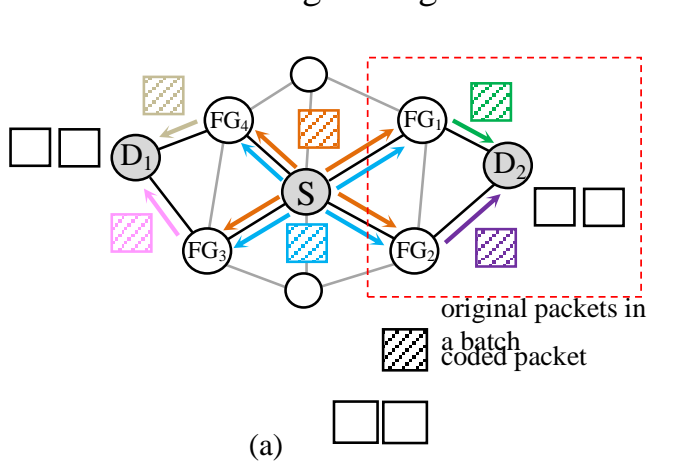

(a)

\section{Figure 4. The Format of (a) Controloverheads and (b) Data Packet in our Proposed Network Coding based Multicast Routing Protocol}

The following flow charts in Pigure 5 show in detail how network coding operates at source node, intermediate nodes, and multicast destination nodes in our proposed network coding based malticast routing protocol.

Next, we will present step-by-step routing and coding algorithm of CRMP with decoding failure awarehess as follows:

- Step 1: Initially, source node sends Join Request (JREQ) packets with its mobility information throughout the networks. Upon receiving JREQ from source node, intermediate nodes will extract mobility information of source node to calculate link lifetime of the wireless link between it and source node. The detailed algorithm and mathematical expression for link's lifetime calculation is presented in detail in [13]. Then, intermediate node updates Node's Mobility and Link's Lifetime in JREQ packet with its mobility information and its link lifetime time, respectively, and forwards this JREQ to other intermediate nodes.

- Step 2: If an intermediate node receives JREQ from previous intermediate node, it performs the same steps as above. If the latest link's lifetime is longer than the previous one stored in its Routing Catch, it will update the Routing Catch before forwarding this JREQ. Otherwise, it does nothing. 


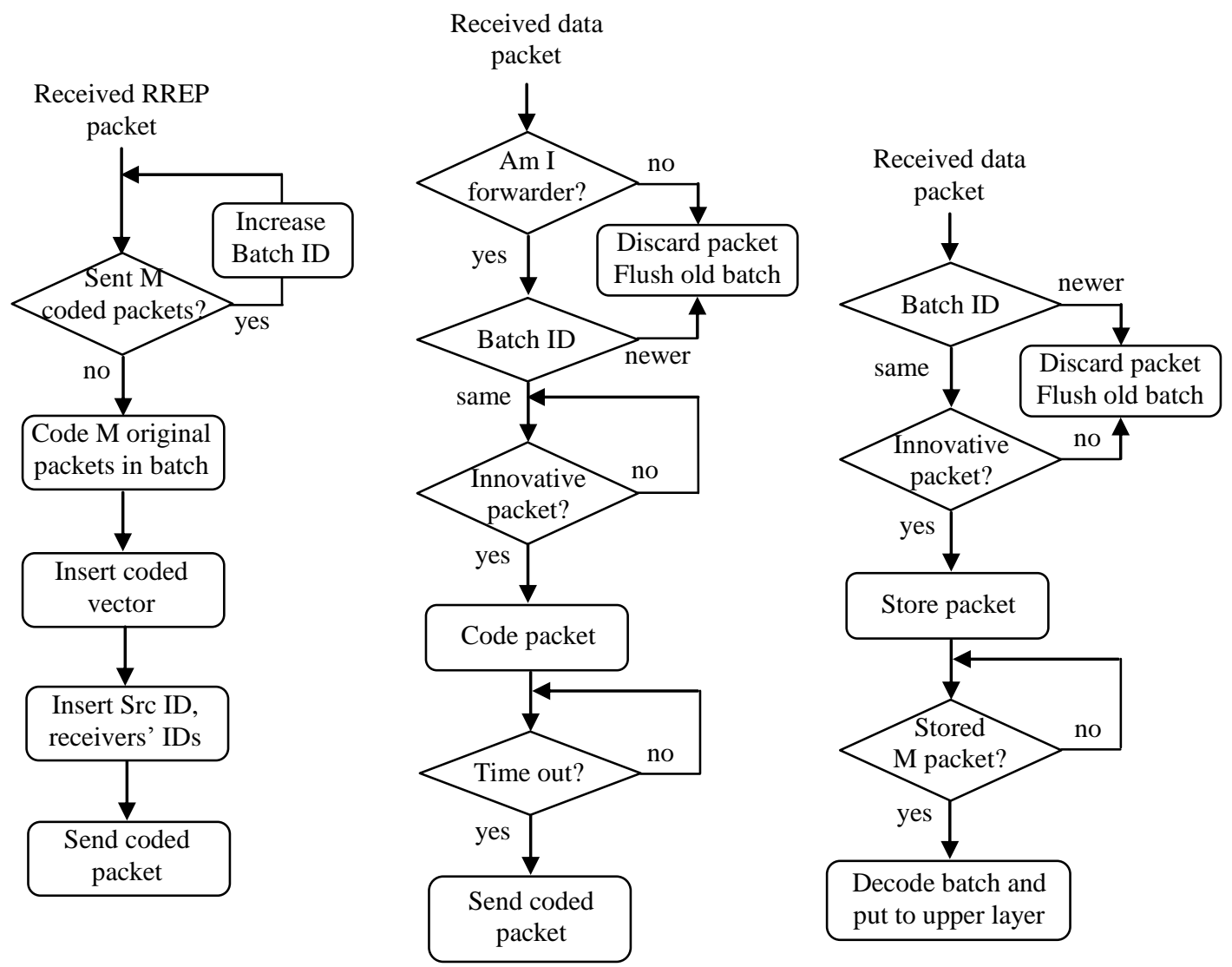

\section{Figure 5. Flow Charts of Codfing Algorithm in our Proposed Network Coding based Múlticast Routing Protocol at (a) Source Node, (b) Intermediate Node, and (c) Multicast Destination Node}

- Step 3: When multicast destination nodes receive JREQ, they will send Join Reply (JREP) to source node ria the most stable paths among available paths. The forwarding nodes on stable paths form robust mesh structure to deliver data packets from source node to multicast destination nodes.

- Step 4: When source node receives JREP packet, it sets route refresh interval based on weakest link information and splits original data packet stream into batches with $\mathrm{M}$ packets in each batch. After sending M packets in a batch, source node increases Batch ID of the next batch. All data packets in a batch will be multiplied with random integer coefficients called elements of code vector to create a coded data packet.

- Step 5: Every time forwarder receives coded data packet, it will check Batch ID in that packet to check whether it is from new bath. If yes, it flushes all packets belonging to old batch in its buffer. Otherwise, it checks if that packet is innovative packet or not. If an innovative packet (i.e., a coded packet having the same Batch ID but linearly independent coding coefficients with previous coded packets) is received, it will be stored in forwarder's buffer to be coded with other packets. If not, it will be discarded. After a specific time, forwarding node will combine coded packets together with random coding coefficients.

- Step 6: When multicast destination nodes receive coded packet, it performs the same steps as forwarder nodes. However, if the number of received coded packets with the same batch size equals to the number written in Batch Size field of data packet, 
destination node will decode those coded packets to retrieve original packets and put them to upper layer. If destination node cannot decode the packet due to lack of coded packets, a process of sending RtE and RtC is triggered to create additional path for delivering coded packets to multicast destination nodes.

\section{Performance Evaluation}

To evaluate the performance of CRMP, we implement CRMP by using OPNET with the following settings: there is one multicast group with one source node and varying number of multicast destination nodes; members join the multicast group at the beginning of simulation and remains as members during the simulation; source hode begins to send coded data packets right after it receives the first JREP packet and continues the sending of coded data packets throughout the simulation; variable number of mobile nodes randomly placed in $1000 \mathrm{~m} \times 1000 \mathrm{~m}$ netyork area:simulation time is $300 \mathrm{~s}$; transmission range is $250 \mathrm{~m}$; 802.11 MAC; data packet size $1 \mathrm{~s} 512$ bytes; data packets are transmitted with constant-based-rate of 20 packets/s; mobile nodes move with Random Waypoint mobility; pause time is andomly selected from $0 \sim 10 \mathrm{~s}$; varying maximum speed $(5,10,20,40,60 \mathrm{~km} / \mathrm{h})$. Each-scenario is simulated 5 times with different node mobility and number of multicast member nodes. The average values are plotted in the graphs.

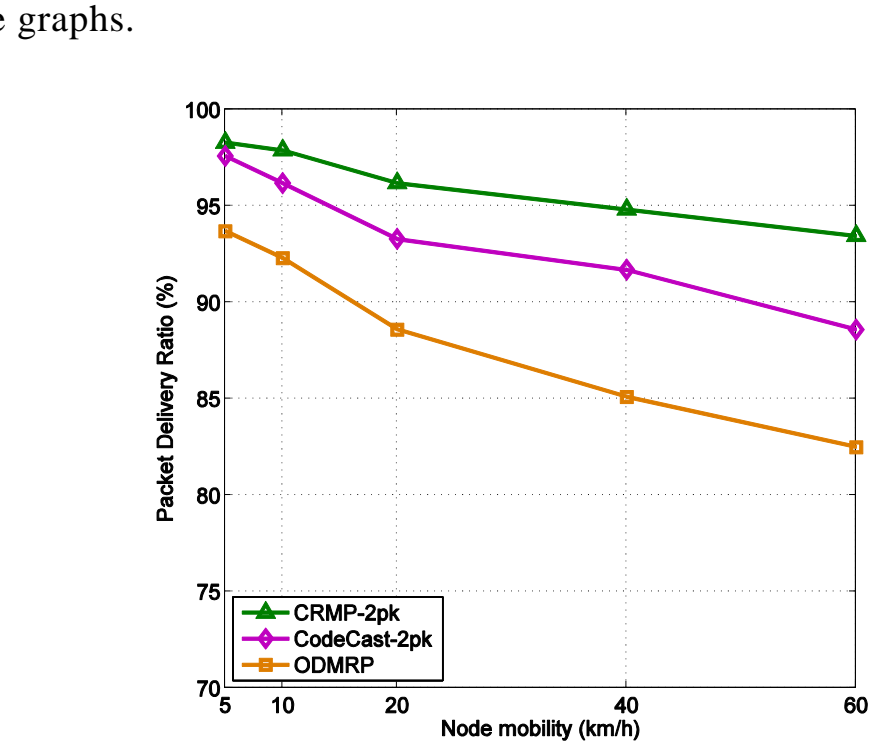

\section{Figure 6. Packet Delivery Ratio (PDR) as a Function of Node Mobility}

Figure 6 shows the packet delivery ratio (PDR), i.e. the ratio of the number of packet received by multicast destination nodes to those generated by source node, as a function of node mobility. Number of multicast member nodes are 3. As we can see in Figure 6, the proposed CRMP has higher PDR than CodeCast and ODMRP, especially when node mobility is high because of enhanced stability of mesh structure and an algorithm to fix decoding failures as mentioned above. 


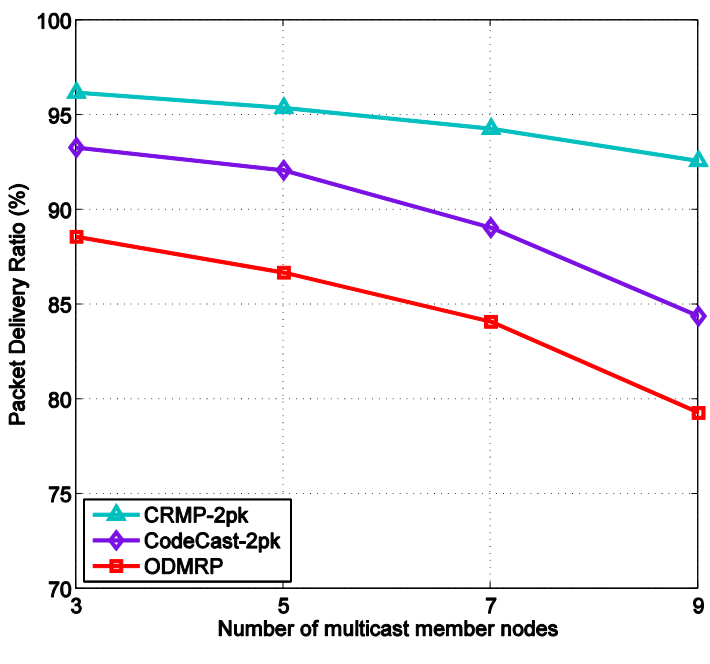

Figure 7. Packet Deivery Ratio (PDR) as a function of the number of multicast member nodes

Figure 7 shows the PDR as a function of the number of multicast member nodes in networks which also refer as the scalability of multicast routing protocols. Node mobility is $40 \mathrm{~km} / \mathrm{h}$. As we can see in Figure 4, CRMP has significantly higher scalability than CodeCast and ODMRP as the number of multicast member nodes increases. The reason is that CRMP uses linear network coding together with optimal route refresh interval to reduce network congestion. CRMP also has a mechanism to prevent decoding failures for all multicast destination nodes.

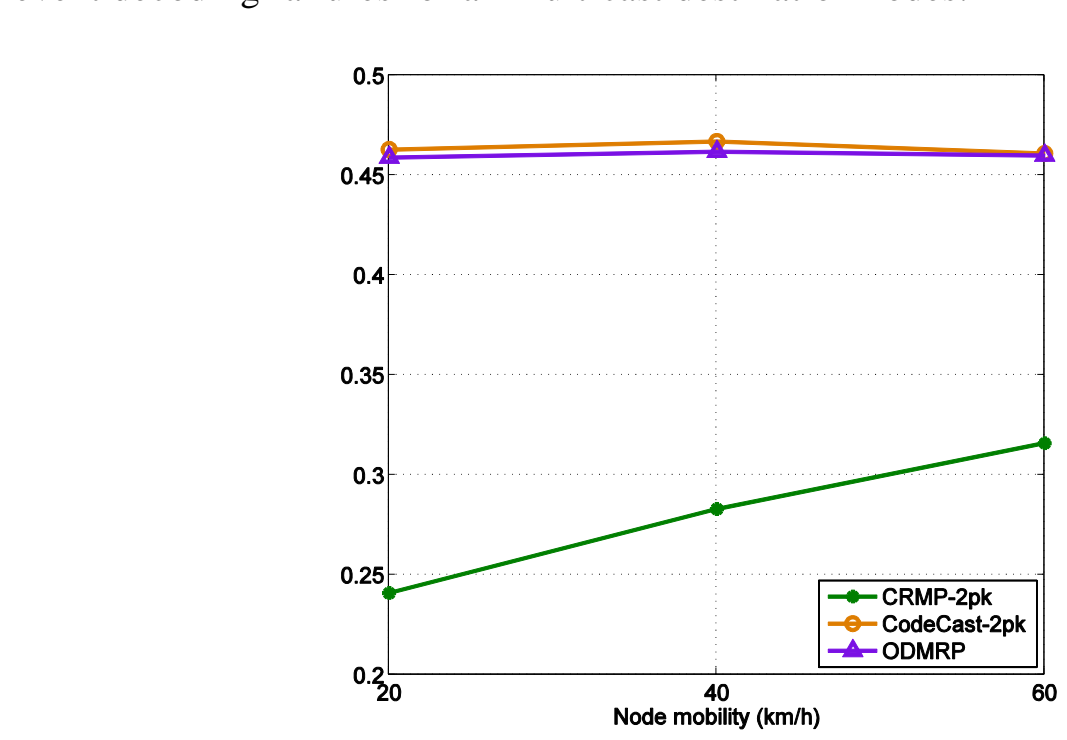

\section{Figure 8. Normalized Number of Control Overheads as a Function of Node Mobility}

Figure 8 shows the normalized number of control overheads as a function of node mobility. The number of multicast member nodes is 3 . The normalized number of control overhead is defined as the ratio of the total number of control overheads, i.e. JREQ, JREP, RtE, RtC, to the number of data 
packets received at multicast receivers. As we can see in Figure 8, since source nodes in ODMRP and CodeCast flood JREQ periodically (every $5 \mathrm{~s}$ in our simulation) to update mesh structure, the normalized number of control overheads is independent of node mobility. Therefore, it may waste network resource when mesh structure is stable when node mobility is low. However, source node in our proposed routing protocol adaptively selects routing update interval to remarkably reduce the number of control overheads, especially JREQs in the network. Although our proposed multicast routing uses additional control overheads, i.e. RtE and RtC, to deal with decoding failure at multicast destination nodes, the normalized value of control overhead is still lower than those of ODMRP and CodeCast.

\section{Figure 9. Normalized Number of Control Overheads as a Function of the Number of Multicast Member Nodes}

Figure 9 shows the normalized number of control overheads as a function the number of multicast member nodes. Node mobility is $40 \mathrm{~km} / \mathrm{h}$. As we can see in Figure 9, normalized number of control overheads decreases when the number of multicast member nodes increase becduse there are more multicast destination nodes in the network. In our proposed multicast routing, additional control overheads, i.e. RtE and $\mathrm{RtC}$, are used to solve decoding failure as multicast destination nodes. Thus, the decreasing rate of the normalized number of control overheads is not as high as those of ODMRP and CodeCast. However, normalized number of control overheads is still lower than those of ODMRP and CodeCast due to the remarkable reduction of the number of JREQs flooding in the network.

Figure 10 shows the drawback of CRMP, the average end-to-end delay. As we can see 1h igure 10, the end-to-end delay of multicast routing protocols with network coding such as CRMP and CodeCast is higher than multicast routing protocol without network coding such as ODMRP. In CRMP, the increase of average end-to-send delay, which also happens in CodeCast, is unavoidable because at each forwarding node it takes time to collect enough appropriate packets to be coded together. However, the gain from the numbers of original packets in coded packet will compensate this drawback as the number of original combined in coded packet increases. 


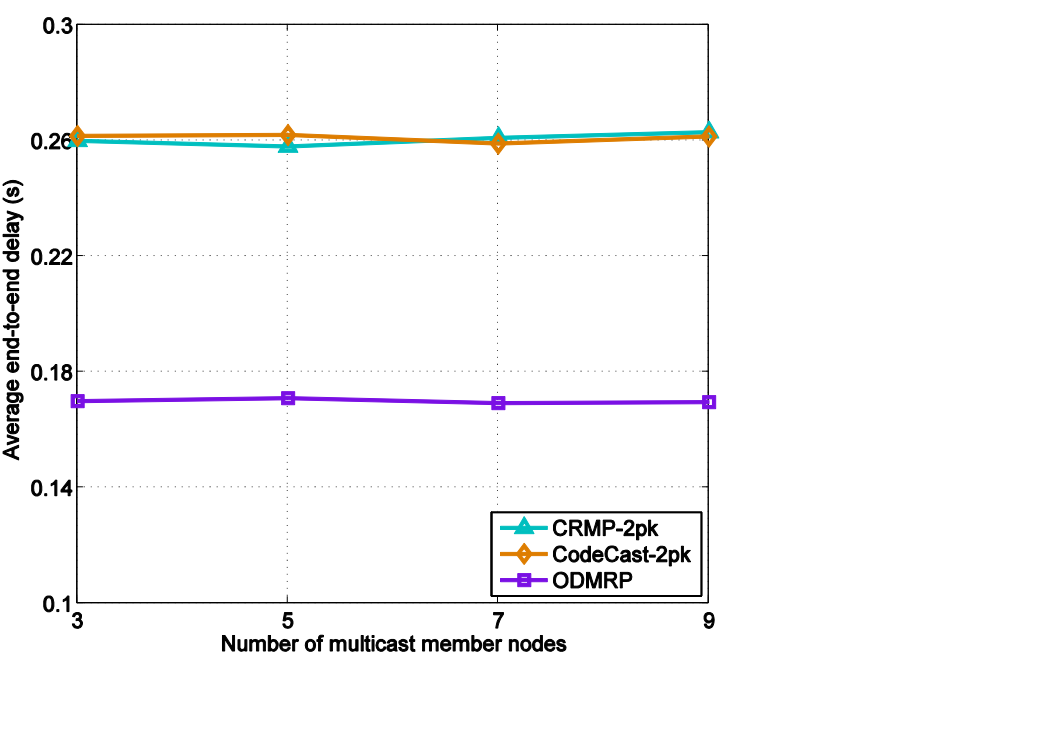

Figure 10. Average End-to-end Delay as a Function of the Number of Multicast Member Nodes

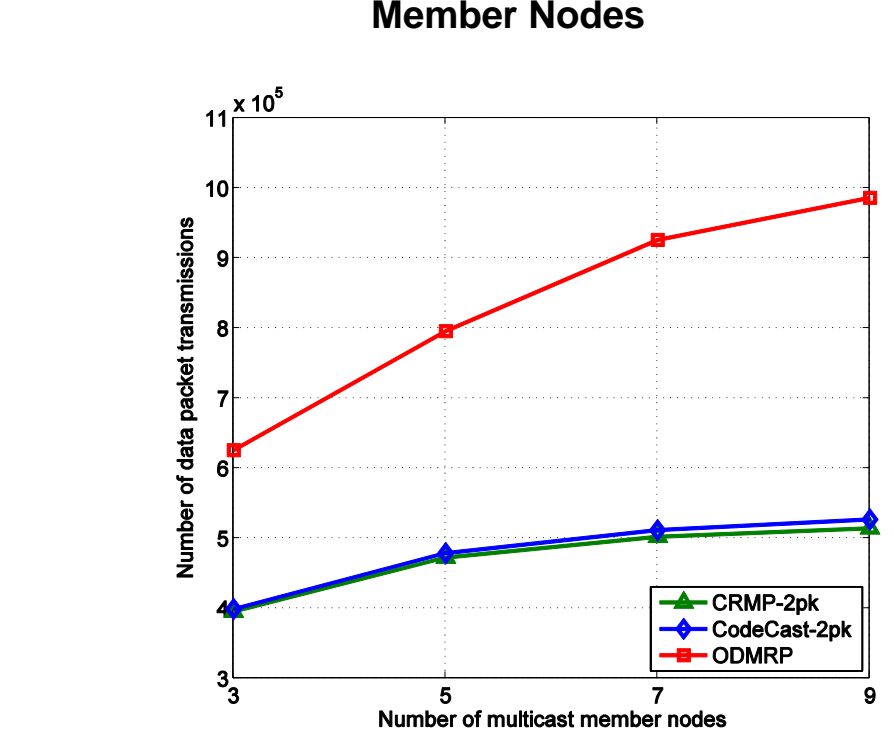

Figurg 11. The Number of Duplicated Data Packet as a Function of the Number of Multicast Member Nodes

Figure 11 shows the number of duplicate data packet as a function of the number of multicast member nodes with node mobility of $20 \mathrm{~km} / \mathrm{h}$. As we can see in Figure 11, CRMP generates significantly lower number of data packet transmissions than ODMRP. As the number of multicast member nodes increases, the gap in the number of duplicate data packet of our proposed CRMP and ODMRP is larger. CRMP has moderately lower number of data transmissions because it can reduce the number of forwarding node by using optimal route refresh interval calculated from the information of node mobility in the network. 


\section{Conclusion}

In this paper, we propose a robust Network Coding based Multicast Routing, named CRMP, in mobile ad-hoc wireless networks. Firstly, a stable mesh topology is established to provide reliable packet delivery from source node to multicast destination nodes in dynamic network topologies as mobile ad-hoc wireless networks. Secondly, we apply linear network coding to forwarders in mesh. By applying linear network coding only to forwarders in mesh topology, we exploit spatial diversity to improve coding and decoding opportunities. Third, each forwarder checks coding and decoding opportunity carefully instead of blindly code packet together. Finally, a local route repairing mechanism is used to prevent multicast destination nodes from decoding failure) The simulation results show that the performance of our proposed network coding based multicast routing protocol outperforms those of CodeCast and ODMRP

\section{Acknowledgements}

This research was supported by Basic Science Research Program through the National Research Foundation of Korea (NRF) funded by the Ministry of Education, Science and Technology (MEST) (2013075605).

\section{References}

[1] R. Ahlswede, N. Cai, S. Li, and R. W, Yeung, "Netwolk Information Flow", IEEE Transactions on Information Theory, vol. 46, no. 4, (2000)

[2] Z. Wang, S. S. Karande, H. R. Sadjadpour and J.J. Garica-Luna-Aceves, O"n the Muticast Capacity of Wireless Ad Hoc Networks with Network Coding", Journal of Communications and Networks, vol. 13, no. 5 (2011).

[3] S. Y. Robert Li, R. W.Yeung, and N. Cai, Kinear Network Coding”, IEEE Transactions on Information, vol. 49, no. 2, (2003).

[4] S. Biswas and R Morris, "ExOR. Opportunistic Multi-Hop Routing for Wireless Networks", Proceeding of ACM SIGCOMM 05 (2005) August 22-26.

[5] S. Chachulski, M. Jennings, S. Katti and Dina Katabi, "Trading Structure for Randomness in Wireless Opportanistic Routing”, Proceeding of ACM SIGCOMM'07, (2007) August 27-31.

[6] S. Katti, H. Rahul, W. Hu, D. Katabi, M. Medard and J. Crowcroft, "XORs in The Air: Practical Wireless Network Coding”, IEEE/ACM Transactions on Network Coding, vol. 16, no. 3, (2008).

[7] J. S. Park, M. Gerla,D Lun, Y. Li and M. Medard, "CodeCast: A Network-Coding-Based Ad Hoc Multicast Protocol”, IEEE N(ireless Communications, vol. 13, (2006).

[8] Y. WenZhong, H.Chuanhe, B. Wang, Z. Zhenyu and W. Tong, "A reliable multicast for MANETs based on opportunistic routing and network coding", Proceeding of 2010 IEEE International Conference on Wireless Communications, Networking and Information Security (WCNIS), (2010) June 2527.

[9] E. E Egbogah, A. O. Fapojuwo and Z. Li, "On the Performance of Network Coding for Multicast Data Deliyery in Large Scale Mobile Ad Hoc Networks", Proceeding of 2010 IEEE 72nd Vehicular Technology Conference Fall (VTC 2010-Fall), (2010) September 6-9.

[10] T. Yang, W. Gang, Z. Qiang, L. Jian and W. Jun, "Improved Network Coding Based on ODMRP Protocol in Ad Hoc Network", Proceeding of International Conference on Advanced Computer Control (ICACC), (2011) January 18-20.

[11] G. Li. Y. Xu, S Li, and D. Cui, “A Multicast Routing Protocol for Network Coding”, Proceeding of 2013 2nd IEEE/CIC International Conference on Communications in China (ICCC), (2013) August 12-14.

[12] S. J. Lee, M. Gearla and C. C. Chiang, “On-Demand Multicast Routing Protocol”, Proceeding of 1999 EEE Wireless Communications and Networking Conference (WCNC), (1999) September 21-24.

[13] L. T. Dung, S. H. Ha and B. An, "A Practical Adaptive Scheme for Enhancing Network Stability in Mobile Ad-hoc Wireless Networks, Lecture Notes in Computer Science (LNCS) 7861, (2013). 


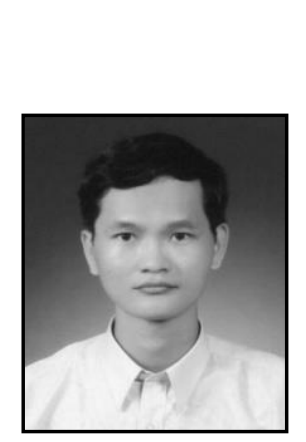

\section{Authors}

Le The Dung, he received the B.S. degree in Electronics and Telecommunication Engineering from Ho Chi Minh City University of Technology, Vietnam, in 2008 and the M.S. degree in Electronics and Computer Engineering from Hongik University, Korea, in 2012. From 2007-2010, we joined Signet Design Solutions Vietnam as hardware designer. He is currently working toward the Ph.D degree in Electronics and Computer Engineering with Hongik University, Korea. His major interests include routing protocols in mobile ad-hoc wireless netyorks and cognitive radio ad-hoc networks, network coding, netyork stability analysis and optimization. He is a student member of the EEE and a student member of the IEIE.

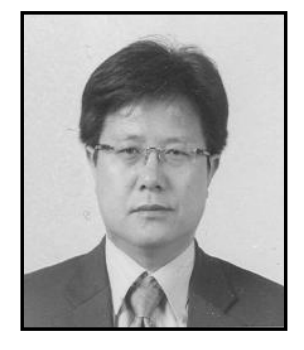

Beongku An, he received he M.S. degree in electrical engineering from the Polytechnic Institute of New York University (Polytechnic University), NY, USA, in 1996 and Ph.D. degree from New Jersey Institute of Technology (NJT), NJ, USA, in 2002, respectively. He received the B.S. degree in electrontc engineering from Kyungpook National University, Korea, in 1988. From 2003, he joined the Faculty of the Department of Computer and Information Communication Engineering, Hongik University in Korea, where he is currently a Professor. From 1990 to 1994 , he was a senior researcher in RIST, Pohang, Rorea. He was Lecturer and RA in NJIT from 1997 to 2002. In 2012, he, worked as a president in IEIE computer society in Korea, and from 2011 to 2014 as a general chair in international conference, ICGHIT, respectively. His current research interests include wireless networks and wireless communications such as ad-hoc networks, sensor network\&, yireless internet, ubiquitous networks, and cellular networks, cognitive radio networks, visible light communications (VLC). Professor An W as listed in Marquis Who's Who in Science and Engineering and Marquis Who in the World, respectively. 